\title{
Role of the Neutrophil-Lymphocyte Count Ratio in the Differential Diagnosis between Pulmonary Tuberculosis and Bacterial Community-Acquired Pneumonia
}

Neul-Bom Yoon, M.D., Choonhee Son, Ph.D., and Soo-Jung Um, M.D.

Division of Respiratory Medicine, Department of Internal Medicine, Dong-A University College of Medicine, Dong-A University Medical Center, Busan, Korea

Background: Differential diagnosis between pulmonary tuberculosis (TB) and bacterial community-acquired pneumonia (CAP) is often challenging. The neutrophil-lymphocyte count ratio (NLR), a convenient marker of inflammation, has been demonstrated to be a useful biomarker for predicting bacteremia. We investigated the usefulness of the NLR for discriminating pulmonary TB from bacterial CAP in an intermediate TB-burden country.

Methods: We retrospectively analyzed the clinical and laboratory characteristics of 206 patients suspected of having pulmonary TB or bacterial CAP from January 2009 to February 2011. The diagnostic ability of the NLR for differential diagnosis was evaluated and compared with that of $\mathrm{C}$-reactive protein.

Results: Serum NLR levels were significantly lower in patients with pulmonary TB than in patients with bacterial CAP $(3.67 \pm 2.12$ vs. $14.64 \pm 9.72, P<0.001)$. A NLR $<7$ was an optimal cut-off value to discriminate patients with pulmonary TB from patients with bacterial CAP (sensitivity $91.1 \%$, specificity $81.9 \%$, positive predictive value $85.7 \%$, negative predictive value $88.5 \%)$. The area under the curve for the NLR $(0.95,95 \%$ confidence interval [Cl], 0.91-0.98) was significantly greater than that of C-reactive protein $(0.83,95 \% \mathrm{Cl}, 0.76$ $0.88 ; P=0.0015$ ).

Conclusions: The NLR obtained at the initial diagnostic stage is a useful laboratory marker to discriminate patients with pulmonary TB from patients with bacterial CAP in an intermediate TB-burden country.

Key Words: C-reactive protein, Lymphocyte, Neutrophil, Pneumonia, Tuberculosis
Received: March 26, 2012

Revision received: October 5, 2012

Accepted: December 14, 2012

Corresponding author: Soo-Jung Um Division of Respiratory Medicine,

Department of Internal Medicine, Dong-A University College of Medicine, Dong-A University Medical Center, 26 Daesingongwon-ro, Seo-gu, Busan 602-715, Korea

Tel: +82-51-240-2769

Fax: +82-51-242-5852

E-mail: sjum@dau.ac.kr (c) The Korean Society for Laboratory Medicine. This is an Open Access article distributed under the terms of the Creative Commons Attribution Non-Commercial License (http://creativecommons.org/licenses/by-nc/3.0) which permits unrestricted non-commercial use, distribution, and reproduction in any medium, provided the original work is properly cited.

\section{INTRODUCTION}

Community-acquired pneumonia (CAP) is one of the most important infectious causes of death in Korea [1]. Early diagnosis and appropriate administration of antibiotics are essential for reducing pneumonia-related morbidity and mortality [2]. Many studies conducted in high-tuberculosis (TB) burden countries have shown that Mycobacterium tuberculosis is a frequent cause of CAP; however, it is frequently difficult to distinguish pulmonary TB from bacterial CAP during the initial diagnostic stage $[3,4]$.
The clinical and radiographic features of TB are often nonspecific, and the sensitivity of microscopic examination of expectorated sputum for acid-fast bacilli is as low as 50-60\% [5].

Delays in diagnosing pulmonary TB can have a negative effect on patient morbidity and mortality. Furthermore, nosocomial transmission of pulmonary TB to other hospitalized patients and healthcare workers can occur. Recently, some studies have suggested that biomarkers such as C-reactive protein (CRP), procalcitonin, and soluble triggering receptor expressed on myeloid cells may play a role in discriminating pulmonary TB from bac- 
terial CAP [6-8]. However, not all of these biomarkers are available in countries with a high TB burden.

The neutrophil-lymphocyte count ratio (NLR) is a readily calculable laboratory marker used to evaluate systemic inflammation. A recent study demonstrated the usefulness of lymphocytopenia for predicting bacteremia by combining conventional infection markers [9]. Additionally, de Jager et al. [10] demonstrated that lymphocytopenia and the NLR were superior to CRP level, and white blood cell (WBC) and neutrophil counts for predicting bacteremia in an emergency care unit.

In this study, we investigated the role of the NLR in discriminating patients with active pulmonary TB from those with bacterial CAP and compared the diagnostic ability of the NLR to that of CRP and WBC.

\section{METHODS}

\section{Patients}

Consecutive adult patients older than $18 \mathrm{yr}$ who were suspected of having CAP from January 2009 to February 2011 were included in this retrospective cohort study. Suspected CAP patients were defined as those with recent-onset respiratory symptoms or signs, abnormal infiltrates on chest radiography, and not recently hospitalized.

Exclusion criteria included use of antibiotics for more than 24 hr at the time of enrollment, conditions known to affect total and differential WBC counts such as hematologic disorders, chronic inflammatory conditions, current steroid therapy, and/or history of steroid use within 3 months before admission, history of chemotherapy or radiotherapy within 4 weeks before enrollment, absence of WBC differential count or CRP data, or change to other diagnosis such as pulmonary embolism, pulmonary edema, or lung cancer during follow-up.

Demographic data were collected and the Pneumonia Patient Outcomes Research Team (PORT) score was calculated [11]. The institutional review board approved this study, and it was conducted in accordance with the Declaration of Helsinki.

\section{Diagnosis of pulmonary TB or bacterial CAP}

Patients were assigned to the pulmonary TB group when 1) $M$. tuberculosis was cultured from sputum or bronchial lavage fluid, or 2) the concentration of adenosine deaminase was $\geq 70$ IU/dL according to lymphocyte-dominant exudative pleural effusion combined with a lung parenchymal lesion suggesting pulmonary TB. Patients were considered to have bacterial CAP when 1 ) clinical signs and new infiltration on chest radiograph were evident and completely resolved after treatment with the appropriate antibiotics, 2) sputum or lavage fluid cultures were M. tuberculosis-negative during clinical follow-up, and 3) viral pathogens were not detected.

For microbiological detection in patients with bacterial CAP, we performed sputum Gram stains, cultures, and blood cultures. Urinary antigen assays were also used to detect Legionella pneumophila and Streptococcus pneumoniae according to clinical indication. In some patients, the virus was isolated from nasopharyngeal secretion specimens using a real-time PCR platform to detect influenza virus A or B, adenovirus, human metapneumovirus, respiratory syncytial virus, rhinovirus, and parainfluenza virus.

\section{Inflammatory markers}

WBC differential count and CRP were determined before antibiotic treatment. Total WBC, neutrophil, and lymphocyte counts were determined using a Sysmex XE-2100 Hematology Analyzer (Sysmex Corp., Kobe, Japan). NLR was defined as absolute neutrophil count divided by absolute lymphocyte count. CRP levels were measured using an automated latex-enhanced turbidimetric immunoassay (Dimension; Dade Behring, Newark, NJ, USA; TBA-200FR; Toshiba, Tokyo, Japan) within 1 hour of sample collection. WBC differential count, NLR, and CRP level were compared between patients with pulmonary TB and patients with bacterial CAP. These inflammatory markers were also compared between patients with pulmonary TB and patients with mild severity pneumonia with a PORT pneumonia severity index (PSI) risk class I or II.

\section{Statistical analysis}

Continuous data are presented as the mean \pm standard deviation. Differences in continuous variables between groups were determined using Student's $t$-test or the Mann-Whitney $U$-test. Categorical data are presented as frequencies. Differences between categorical variables were analyzed using Pearson's chisquare test or Fisher's exact test. Correlation between NLR and CRP was analyzed using Spearman's rank correlation test. ROC curve analysis was performed to identify the most useful cut-off levels for NLR, CRP, WBC count, neutrophil count, and lymphocyte count to identify the greatest sum of sensitivity and specificity for distinguishing pulmonary TB from bacterial CAP. The ability of NLR, CRP, WBC count, neutrophil count, and lymphocyte count to distinguish pulmonary TB from bacterial CAP was compared using the area under the curve (AUC). A $P$ value $\leq 0.05$ was considered statistically significant. All statistical anal- 
yses were performed using SPSS 19.0 (SPSS Inc., Chicago, IL, USA).

\section{RESULTS}

\section{Patients}

We enrolled 206 patients in this study (Fig. 1), of which 112 (54.4\%) had pulmonary TB and 94 (45.6\%) had bacterial CAP. The median age of patients with pulmonary TB and bacterial CAP was 54 yr (range, 20-86 yr) and $70 \mathrm{yr}$ (range, 18-86 yr), respectively. Baseline clinical characteristics are listed in Table 1.

Among the patients with pulmonary TB, 98 (87.5\%) had $M$. tuberculosis culture-positive respiratory specimens. The remaining patients had pleural effusion and lung infiltration on radiography compatible with TB. Treatment failure occurred in one patient.

The causative pathogens were determined in 27 patients (28.7\%) with bacterial CAP. Streptococcus pneumoniae was detected in 17 patients using urinary antigen tests, respiratory specimen culture, or blood culture. Other pathogens identified were Klebsiella pneumonia $(\mathrm{N}=4)$, Escherichia coli $(\mathrm{N}=2)$, methicillinsensitive Staphylococcus aureus $(\mathrm{N}=1)$, methicillin-resistant $S$. aureus $(\mathrm{N}=1)$, Acinetobacter baumannii $(\mathrm{N}=1)$, and Stenotrophomonas maltophila $(\mathrm{N}=1)$. The mean PSI was 111.03. Seventy-four patients $(78.7 \%)$ were categorized as greater than PSI

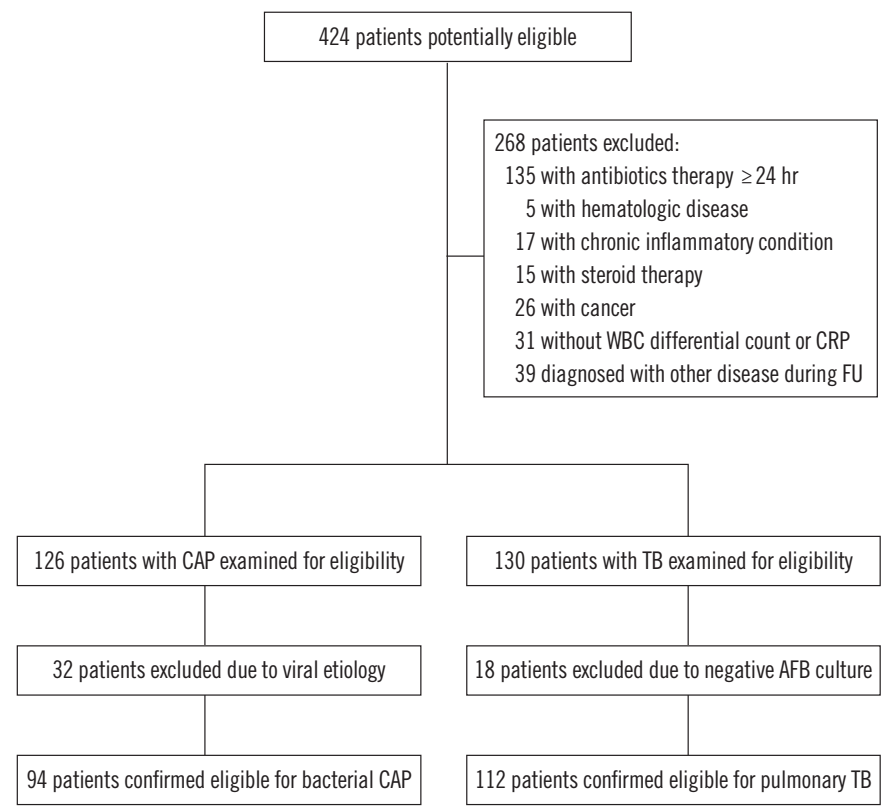

Fig. 1. Patient flow chart.

Abbreviations: WBC, white blood cell; CRP, C-reactive protein; FU, follow up; CAP, community-acquired pneumonia; TB, tuberculosis; AFB, acidfastbacilli. risk class III. Twenty patients (21.3\%) were regarded as having mild-severity pneumonia. In-hospital mortality for the CAP group was $12.8 \%(\mathrm{~N}=12)$.

\section{Inflammation markers}

Mean and median values of the NLR were $9.69 \pm 9.02$ and 6.36 , respectively. Total WBC and neutrophil count, NLR, and CRP were significantly lower in patients with pulmonary TB than in patients with bacterial CAP, whereas lymphocyte count was significantly higher in patients with pulmonary TB than in patients with bacterial CAP (Table 2).

We conducted a subgroup analysis of patients with mild pneumonia ( $\mathrm{N}=20$, PSI risk classes I and II) and patients with pulmonary TB to assess the diagnostic ability of the NLR. Total WBC count (7.19 \pm 2.48 vs. $12.59 \pm 5.41, P<0.001$ ), neutrophil count ( $4.79 \pm 2.14$ vs. $10.38 \pm 4.78, P<0.001)$, NLR ( $3.67 \pm 2.12$ vs. 9.65 $\pm 6.41, P<0.001)$, and CRP $(6.57 \pm 7.31$ vs. $17.48 \pm 10.76, P<$ 0.001 ) were also significantly lower in patients with pulmonary TB than in patients with mild-severity pneumonia. However, lym-

Table 1. Baseline clinical characteristics of the study population

\begin{tabular}{lccr}
\hline & $\begin{array}{c}\text { Bacterial CAP } \\
(\mathrm{N}=94)\end{array}$ & $\begin{array}{c}\text { Tuberculosis } \\
(\mathrm{N}=112)\end{array}$ & P value \\
\hline Age (yr) & $70(18-86)$ & $54(20-86)$ & $<0.001$ \\
Male & $61(64.9)$ & $66(58.9)$ & 0.392 \\
History of tuberculosis & $24(25.5)$ & $26(23.2)$ & 0.746 \\
Symptom duration $>2$ weeks & $9(9.6)$ & $68(60.7)$ & $<0.001$ \\
Hemoptysis & $7(7.4)$ & $21(18.8)$ & 0.024 \\
Fever & $87(92.6)$ & $48(42.9)$ & $<0.001$ \\
Cough & $71(75.5)$ & $98(87.5)$ & 0.030 \\
Night sweats & $15(16.0)$ & $34(30.4)$ & 0.021 \\
Weight loss & $2(2.1)$ & $31(27.7)$ & $<0.001$ \\
\hline
\end{tabular}

Values are presented as median (range) or number (percentage). Abbreviation: CAP, community-acquired pneumonia.

Table 2. Baseline laboratory characteristics of the study population

\begin{tabular}{lccc}
\hline & $\begin{array}{c}\text { Bacterial CAP } \\
(\mathrm{N}=94)\end{array}$ & $\begin{array}{c}\text { Tuberculosis } \\
(\mathrm{N}=112)\end{array}$ & Pvalue \\
\hline WBC $\left(10^{\circ} / \mathrm{L}\right)$ & $14.28 \pm 6.73$ & $7.19 \pm 2.48$ & $<0.001$ \\
Neutrophil $\left(10^{9} / \mathrm{L}\right)$ & $12.44 \pm 6.38$ & $4.79 \pm 2.14$ & $<0.001$ \\
Lymphocyte $\left(10^{9} / \mathrm{L}\right)$ & $1.01 \pm 0.54$ & $1.51 \pm 0.60$ & $<0.001$ \\
NLR & $14.64 \pm 9.72$ & $3.67 \pm 2.12$ & $<0.001$ \\
CRP $(\mathrm{mg} / \mathrm{dL})$ & $18.23 \pm 10.64$ & $6.57 \pm 7.31$ & $<0.001$ \\
\hline
\end{tabular}

Values are presented as mean \pm standard deviation.

Abbreviations: CAP, community-acquired pneumonia; WBC, white blood cell; $\mathrm{NLR}$, neutrophil-lymphocyte count ratio; CRP, C-reactive protein. 
Table 3. Diagnostic validity of NLR, CRP, and WBC with differential counts in differentiating pulmonary TB from bacterial CAP

\begin{tabular}{lccccc}
\hline & Sensitivity & Specificity & PPV & NPV & Accuracy \\
\hline NLR $^{*}$ & 91.1 & 81.9 & 85.7 & 88.5 & 86.9 \\
CRP $^{\dagger}$ & 67.5 & 85.1 & 79.4 & 75.5 & 77.1 \\
WBC count $^{\ddagger}$ & 90.2 & 77.7 & 82.1 & 86.7 & 84.0 \\
Neutrophil count $^{5}$ & 90.2 & 80.9 & 84.9 & 87.3 & 85.9 \\
Lymphocyte count" $^{\|}$ & 82.1 & 61.7 & 71.9 & 74.4 & 72.8
\end{tabular}

Values are presented as percentages.

Cut-off values for each parameter: $\mathrm{NLR}^{*}<7.0 ; \mathrm{CRP}^{\dagger}<7.0 \mathrm{mg} / \mathrm{dL} ; \mathrm{WBC}$ $<10.100 \times 10^{9} / \mathrm{L}^{\text {; neutrophil }}{ }^{\S}<7.740 \times 10^{9} / \mathrm{L}^{\text {; lymphocyte }}$ ly $^{\prime \prime}>1.001 \times 10^{9} / \mathrm{L}$. Abbreviations: TB, tuberculosis; CAP, community-acquired pneumonia; NLR, neutrophil-lymphocyte count ratio; CRP, C-reactive protein; WBC, white blood cell; PPV, positive predictive value; NPV, negative predictive value.

phocyte count was not significantly different between groups.

The strongest correlation was noted between NLR and neutrophil count $(r=0.832, P=0.01)$. A positive correlation was also detected between NLR and CRP $(r=0.562, P=0.01)$ as well as between NLR and WBC count $(r=0.738, P=0.01)$. A negative correlation was identified between NLR and lymphocyte count $(r=-0.644, P=0.01)$.

\section{Diagnostic accuracy for discriminating pulmonary TB from bacterial CAP}

A NLR $<7.0$ was identified as the optimal cut-off value for discriminating patients with pulmonary TB from patients with bacterial CAP, yielding $91.1 \%$ sensitivity, $81.9 \%$ specificity, $85.7 \%$ positive predictive value, and $88.5 \%$ negative predictive value (Table 3). The cut-off value for CRP was less than $7.0 \mathrm{mg} / \mathrm{dL}$. Using this cut-off value, CRP showed $67.5 \%$ sensitivity and $85.1 \%$ specificity, $79.4 \%$ positive predictive value, and $75.5 \%$ negative predictive value. The NLR AUC (AUC, 0.93; 95\% confidence interval [Cl], 0.88-0.96) was significantly greater than that of CRP (AUC, 0.83; 95\% Cl, 0.76-0.88; $P=0.0015$ ) (Fig. 2). The NLR AUC was also significantly greater than that of WBC count (AUC, 0.86; 95\% Cl, 0.81-0.91; $P=0.0016$ ), neutrophil count (AUC, 0.89; $95 \% \mathrm{Cl}, 0.84-0.93 ; P=0.0197$ ), and lymphocyte count (AUC, $0.74 ; 95 \% \mathrm{Cl}, 0.68-0.80 ; P<0.0001)$. In patients with mild pneumonia, the NLR AUC (AUC, 0.87; 95\% Cl, 0.76-0.92) was significantly greater than that of lymphocyte count (AUC, $0.61 ; 95 \% \mathrm{Cl}$, 0.52-0.69; $P=0.0019$ ). However, there was no significant difference in the AUC between NLR and CRP (AUC, $0.81 ; 95 \% \mathrm{Cl}$, $0.72-0.88 ; P=0.5721$ ), NLR and WBC count (AUC, $0.81 ; 95 \%$ $\mathrm{Cl}, 0.73-0.87 ; P=0.3523$ ), and NLR and neutrophil count (AUC, $0.86 ; 95 \% \mathrm{Cl}, 0.79-0.91 ; P=0.8466)$.

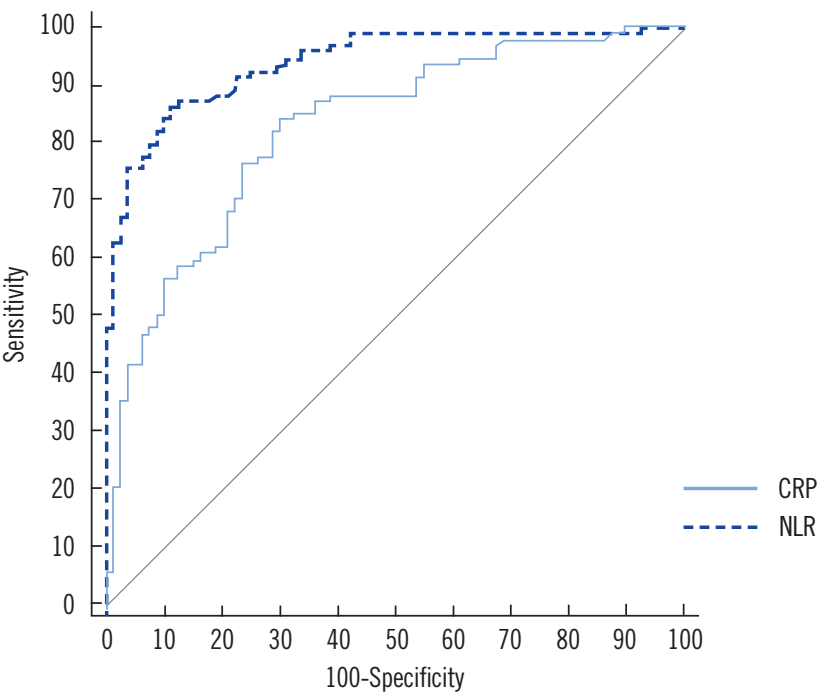

Fig. 2. ROC curves of C-reactive protein (CRP) and neutrophil-lymphocyte count ratio (NLR) for discriminating tuberculosis from bacterial community-acquired pneumonia. The area under the curve for NLR (AUC, 0.93; 95\% confidence interval [CI], 0.88-0.96) was significantly greater than that for CRP (AUC, 0.83; 95\% Cl, 0.760.88) $(P=0.0015)$

\section{DISCUSSION}

In this study of consecutive patients recruited from clinical settings, the NLR was a useful laboratory marker for discriminating between patients with pulmonary TB and other bacterial CAP at the initial diagnostic stage for intermediate TB prevalence. The findings of this study included the following: 1) Serum NLR levels were significantly lower in patients with pulmonary $T B$ than in patients with bacterial CAP; 2) A NLR $<7$ was the optimal cutoff value for discriminating patients with pulmonary TB from patients with bacterial CAP; 3) The diagnostic ability of the NLR was superior to that of CRP, WBC count, neutrophil count, and lymphocyte count for discriminating patients with pulmonary TB from patients with bacterial CAP. This is the first study to demonstrate the diagnostic ability of the NLR for differentiating between pulmonary TB and bacterial CAP.

Korea is an intermediate TB-burden country, with approximately 43,000 cases of pulmonary TB newly diagnosed in 2009 [12]. The frequency of $M$. tuberculosis identification in patients with suspected CAP is as high as $12 \%$ in Hong Kong [4] and $21 \%$ in Singapore [13]; the prevalence of TB in these countries is similar to that in Korea. Distinguishing pulmonary TB from CAP is important in public health; immediate isolation and early treatment with anti-TB agents is essential for patients with pulmonary TB. However, differentiating between pulmonary TB 
and CAP based only on history, physical examination, and radiological findings is frequently difficult. Therefore, a rapid and readily available test to distinguish pulmonary TB from CAP is necessary.

The physiological immune responses of circulating leukocytes to various stressful events are characterized by an increased neutrophil count and decreased lymphocyte count. An increase in total WBC and neutrophils is an inflammatory reaction, particularly when caused by a bacterial infection [14]. Lymphocytopenia has also been described as a diagnostic marker of bacterial infection [9]. Therefore, the NLR is thought to have stronger discriminative power for predicting bacteremia compared to discrimination based on neutrophilia or lymphocytopenia alone. Goodman et al. [15] initially suggested the usefulness of the NLR for diagnosing patients with suspected appendicitis. In their study, the NLR was a more sensitive parameter of bacterial infection than an increased WBC count. Zahorec et al. [14] carried out a prospective longitudinal observational study, in which they reported a correlation between clinical course severity and lymphocytopenia in patients with severe sepsis and septic shock in an oncologic intensive care unit. They proposed using the NLR as an additional marker of infection in the intensive care unit [14]. Recently, de Jager et al. [10] demonstrated that both lymphocytopenia and the NLR were better predictors of bacteremia than routine parameters such as CRP level, WBC count, and neutrophil count in an emergency care unit. Currently, the NLR has been garnering interest as a survival predictor in various clinical situations, ranging from oncological to cardiovascular diseases [16-19].

The NLR of pleural fluid has been evaluated as an adjunctive diagnostic tool in patients with TB pleurisy [20, 21]. CD4+ T lymphocytes and macrophages are essential in the pathogenesis of TB since cell-mediated immunity is involved. In contrast, neutrophilia is the primary cellular phenomenon during development of bacterial CAP. Therefore, the NLR of blood samples may be a good laboratory marker for discriminating patients with pulmonary TB from patients with bacterial CAP.

In this study, total WBC with differential count, CRP, and NLR were significantly different between the pulmonary TB group and the bacterial CAP group. The NLR was the most powerful discriminative marker, as evidenced by its high AUC. Neutrophil count was the second best discriminative marker, followed by WBC count, CRP, and lymphocyte count. Notably, easily evaluable complete blood count parameters such as total WBC and neutrophil counts also demonstrated considerable discriminative power, although less than that of the NLR. Moreover, their sensitivities, specificities, and accuracies were relatively high. The NLR can be easily determined from a routine laboratory exam, and this simple ratio is available in most institutions. Furthermore, the NLR is an easier and less expensive method compared to other inflammatory biomarkers such as CRP, procalcitonin, and soluble triggering receptor expressed on myeloid cells, which recent studies have suggested as differential diagnostic markers [6-8].

However, there are several limitations in this study. First, it was retrospective, nonrandomized, and conducted in a single center; thus, it was subject to selection bias. Investigations involving a larger sample size and prospective analysis are necessary. Second, CAP-causing pathogens could only be identified in a minority of patients. However, CAP causative pathogens are not clearly defined [22, 23]. Third, other inflammatory markers such as procalcitonin were not included and compared with the NLR in the current analysis. However, procalcitonin is an expensive exam that is not available in all hospitals.

In conclusion, the current study showed that the NLR derived from a single blood sample during the initial diagnostic stage is a very useful laboratory marker for discriminating patients with pulmonary TB from patients with bacterial CAP. The diagnostic ability of the NLR is superior to that of CRP, and WBC, neutrophil, and lymphocyte counts. Therefore, the NLR should be incorporated into routine exams in patients with an ambiguous diagnosis between pulmonary TB and bacterial CAP.

\section{Authors' Disclosures of Potential Conflicts of Interest}

No potential conflicts of interest relevant to this article were reported.

\section{Acknowledgement}

This study was supported by research funds from Dong-A University.

\section{REFERENCES}

1. http://www.kostat.go.kr/portal/korea/kor_ki/1/1/index.action?bmode= read\&cd=S004001 (Updated on Oct 2010)

2. Marrie TJ. Community-acquired pneumonia. Clin Infect Dis 1994;18: 501-13.

3. Liam CK, Pang YK, Poosparajah S. Pulmonary tuberculosis presenting as community-acquired pneumonia. Respirology 2006;11:786-92.

4. Chan $\mathrm{CH}$, Cohen M, Pang J. A prospective study of community-acquired pneumonia in Hong Kong. Chest 1992;101:442-6. 
5. Siddiqi K, Lambert ML, Walley J. Clinical diagnosis of smear-negative pulmonary tuberculosis in low-income countries: the current evidence. Lancet Infect Dis 2003;3:288-96.

6. Ugajin M, Miwa S, Shirai M, Ohba H, Eifuku T, Nakamura H, et al. Usefulness of serum procalcitonin level in pulmonary tuberculosis. Eur Respir J 2011;37:371-5.

7. Kang YA, Kwon SY, Yoon HI, Lee JH, Lee CT. Role of C-reactive protein and procalcitonin in differentiation of tuberculosis from bacterial community acquired pneumonia. Korean J Intern Med 2009;24:337-42.

8. Tintinger GR, van der Merwe JJ, Fickl H, Rheeder P, Feldman C, Anderson R. Soluble triggering receptor expressed on myeloid cells in sputum of patients with community-acquired pneumonia or pulmonary tuberculosis: a pilot study. Eur J Clin Microbiol Infect Dis 2012;31:73-6.

9. Wyllie DH, Bowler IC, Peto TE. Relation between lymphopenia and bacteraemia in UK adults with medical emergencies. J Clin Pathol 2004; 57:950-5.

10. de Jager CP, van Wijk PT, Mathoera RB, de Jongh-Leuvenink J, van der Poll T, Wever PC. Lymphocytopenia and neutrophil-lymphocyte count ratio predict bacteremia better than conventional infection markers in an emergency care unit. Crit Care 2010;14:R192.

11. Fine MJ, Auble TE, Yealy DM, Hanusa BH, Weissfeld LA, Singer DE, et al. A prediction rule to identify low-risk patients with community-acquired pneumonia. N Engl J Med 1997;336:243-50.

12. WHO, Global Tuberculosis Control 2011. http://www.who.int/tb/publications/global_report/2011/gtbr11_full.pdf (Updated on Oct 2011)

13. Hui KP, Chin NK, Chow K, Brownlee A, Yeo TC, Kumarasinghe G, et al. Prospective study of the aetiology of adult community acquired bacterial pneumonia needing hospitalisation in Singapore. Singapore Med J 1993; 34:329-34.

14. Zahorec R. Ratio of neutrophil to lymphocyte counts--rapid and simple parameter of systemic inflammation and stress in critically ill. Bratisl Lek Listy 2001;102:5-14.
15. Goodman DA, Goodman CB, Monk JS. Use of the neutrophil: Iymphocyte ratio in the diagnosis of appendicitis. Am Surg 1995;61:257-9.

16. Gibson PH, Croal BL, Cuthbertson BH, Small GR, Ifezulike Al, Gibson G, et al. Preoperative neutrophil-lymphocyte ratio and outcome from coronary artery bypass grafting. Am Heart J 2007;154:995-1002.

17. Halazun KJ, Aldoori A, Malik HZ, Al-Mukhtar A, Prasad KR, Toogood GJ, et al. Elevated preoperative neutrophil to lymphocyte ratio predicts survival following hepatic resection for colorectal liver metastases. Eur J Surg Oncol 2008;34:55-60.

18. Tamhane UU, Aneja S, Montgomery D, Rogers EK, Eagle KA, Gurm HS. Association between admission neutrophil to lymphocyte ratio and outcomes in patients with acute coronary syndrome. Am J Cardiol 2008; 102:653-7.

19. Sarraf KM, Belcher E, Raevsky E, Nicholson AG, Goldstraw P, Lim E. Neutrophil/lymphocyte ratio and its association with survival after complete resection in non-small cell lung cancer. J Thorac Cardiovasc Surg 2009; 137:425-8.

20. Rokayan SA. Serum adenosine deaminase activity and its isoenzyme in patients treated for tuberculosis. J Coll Physicians Surg Pak 2003;13:11-4.

21. Burgess LJ, Maritz FJ, Irene Le Roux I, Taljaard JJ. Combined use of pleural adenosine deaminase with lymphocyte/neutrophil ratio. Increased specificity for the diagnosis of tuberculous pleuritis. Chest 1996;109: 414-9.

22. Charles PG, Whitby M, Fuller AJ, Stirling R, Wright AA, Korman TM, et al. The etiology of community-acquired pneumonia in Australia: why penicillin plus doxycycline or a macrolide is the most appropriate therapy. Clin Infect Dis 2008;46:1513-21.

23. Song JH, Oh WS, Kang Cl, Chung DR, Peck KR, Ko KS, et al. Epidemiology and clinical outcomes of community-acquired pneumonia in adult patients in Asian countries: a prospective study by the Asian network for surveillance of resistant pathogens. Int J Antimicrob Agents 2008;31: 107-14. 